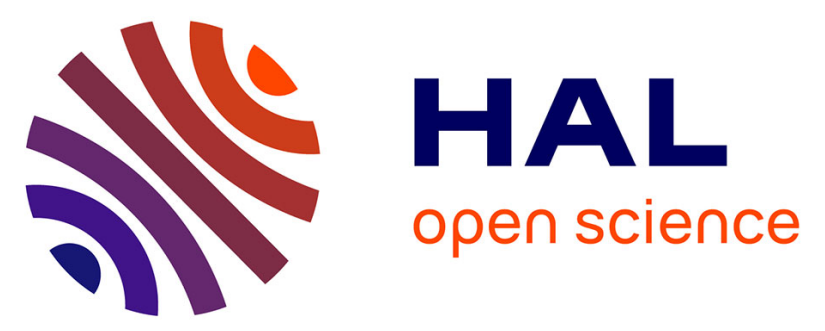

\title{
Identification of compounds active against quiescent artemisinin-resistant Plasmodium falciparum parasites via the quiescent-stage survival assay (QSA)
}

Thibaud Reyser, Lucie Paloque, Manel Ouji, Michel Nguyen, Sandie Ménard, Benoit Witkowski, Jean-Michel Augereau, Françoise Benoit-Vical

\section{To cite this version:}

Thibaud Reyser, Lucie Paloque, Manel Ouji, Michel Nguyen, Sandie Ménard, et al.. Identification of compounds active against quiescent artemisinin-resistant Plasmodium falciparum parasites via the quiescent-stage survival assay (QSA). Journal of Antimicrobial Chemotherapy, 2020, 75 (10), pp.28262834. 10.1093/jac/dkaa250 . hal-02948398

\section{HAL Id: hal-02948398 \\ https://hal.science/hal-02948398}

Submitted on 6 Oct 2021

HAL is a multi-disciplinary open access archive for the deposit and dissemination of scientific research documents, whether they are published or not. The documents may come from teaching and research institutions in France or abroad, or from public or private research centers.
L'archive ouverte pluridisciplinaire HAL, est destinée au dépôt et à la diffusion de documents scientifiques de niveau recherche, publiés ou non, émanant des établissements d'enseignement et de recherche français ou étrangers, des laboratoires publics ou privés. 

.
Identification of compounds active against quiescent artemisinin-resistant Plasmodium falciparum parasites via the Quiescent-stage Survival Assay

Thibaud REYSER ${ }^{1,2, \$}$, Lucie PALOQUE ${ }^{1,2, \$, \ddagger}$, Manel OUJI ${ }^{1,2}$, Michel NGUYEN ${ }^{1,2}$, Sandie MENARD ${ }^{3}$, Benoit WITKOWSKI ${ }^{4}$, Jean-Michel AUGEREAU ${ }^{1,2}$, Françoise BENOIT-VICAL ${ }^{1,2,5, \text { * }^{*}}$

(1)

${ }^{1}$ LCC-CNRS, Laboratoire de Chimie de Coordination, Université de Toulouse, CNRS,

Toulouse, France.

${ }^{2}$ Institut de Pharmacologie et de Biologie Structurale, IPBS, Université de Toulouse, CNRS, UPS, France.

${ }^{3}$ Present adress: CPTP, Centre de Physiopathologie de Toulouse Purpan, INSERM U1043, CNRS UMR5282, Université de Toulouse, Toulouse, France

${ }^{4}$ Malaria Molecular Epidemiology Unit, Pasteur Institute in Cambodia, Phnom Penh, Cambodia.

${ }^{5}$ INSERM, Institut National de la Santé et de la Recherche Scientifique, France

Running title: Compounds active against quiescent artemisinin-resistant malaria parasites

* Author for correspondence: Francoise.Vical@inserm.fr

CNRS, LCC (Laboratoire de Chimie de Coordination), 205 route de Narbonne, BP 44099, F31077 Toulouse Cedex 4, France. Phone: +00 33561175823

${ }^{\$}$ These authors contributed equally to this work. ${ }^{\ddagger}$ LP and FBV share senior authorship. 
ABSTRACT

Background: Quiescence is an unconventional mechanism of Plasmodium survival mediating artemisinin resistance. This phenomenon increases the risks of clinical failures following Artemisinin-based Combination Therapies (ACTs) by slowing parasite clearance and allowing the selection of parasites resistant to partner drugs.

Objectives: To thwart this multi-resistance, the quiescent state of artemisinin-resistant parasites must be taken into consideration from the very early stages of the drug discovery process. Methods: We designed a novel phenotypic assay named Quiescent-stage Survival Assay (QSA) to assess the antiplasmodial activity of drugs on quiescent parasites. This assay was first validated on quiescent forms from different artemisinin-resistant parasite lines (laboratory strain and field isolates), using two reference drugs with different mechanisms of action: chloroquine and atovaquone. Furthermore, the efficacies of different partner drugs of artemisinins used in ACTs were investigated against both laboratory strains and field isolates from Cambodia.

Results: Our results highlight that because of quiescence-based mechanism and the respective pharmacological targets of drugs, their efficacies on artemisinin-resistant parasites may be different between quiescent parasites and their proliferating forms.

Conclusions: These data confirm the high relevance of adding the chemosensitivity evaluation of quiescent parasites by the specific in vitro QSA to the antiplasmodial drug development process in the current worrisome context of artemisinin resistance. 


\section{INTRODUCTION}

Artemisinin-based combination therapies (ACTs), using artemisinin or its derivatives (ARTs) combined with other antiplasmodial drugs, are recommended for the treatment of Plasmodium falciparum malaria. ARTs produce a fast reduction in the parasite numbers, leaving the remaining parasites exposed to the long-lasting partner drug. ${ }^{1}$ The widespread use of ACTs has significantly contributed to the historical decrease of malaria mortality observed over the last 15 years in all endemic countries. ${ }^{2}$ However, Plasmodium resistance to ARTs emerged in 2006 and could now jeopardize malaria elimination programs. ${ }^{3-7}$ ARTs-resistance is linked to a mutation on the kelch13 gene, ${ }^{8}$ which encodes for a protein involved in different mechanisms, among which hemoglobin uptake. ${ }^{9}$ ARTs-resistance is mediated by a quiescent state upon ARTs exposure, ${ }^{10}$ in a mechanism that dramatically differs from other antimalarial drug resistance mechanisms. This parasite cell cycle arrest is characterized by a drastic downregulation of the overall metabolism to allow parasites to face ARTs-induced cellular damage, ${ }^{11,12}$ and subsequent up-regulation to enable parasite recrudescence after drug elimination. ${ }^{10,13}$ In the field, ACT-resistance is always associated with ARTs-resistance. Either ARTs-resistance facilitates emergence of ACT-resistance by allowing partner drug resistance selection in artemisinin resistant parasites or ACT-resistance is acquired after recombination between ARTs-resistant and partner drug-resistant parasite lineages. ${ }^{7,14-19}$ Long-term artemisinin drug pressure also triggers in vitro acquisition of a multidrug tolerance phenotype based on the extended capacity of ARTs-resistant parasites to survive other antiplasmodial drugs through a quiescence mechanism. ${ }^{20}$ Extending ACT treatment from 2 to 3 days in order to improve clinical efficacy thus increases artemisinin drug pressure, leading to a higher frequency of $P$. falciparum ARTs-resistance cases. ${ }^{21,22}$ During ACT treatment, due to the respective pharmacokinetics properties of both drugs, parasites are first exposed to ARTs. That 
is why, in an ARTs-resistance scenario, ring-stage parasites first enter a quiescent state and are then exposed to partner drugs. Therefore, a partner drug that is active against quiescent parasites should be the gold standard for malaria eradication policies and programs. Because standard chemo-sensitivity assays have shown their inability to study artemisinin resistance, ${ }^{8,10,20}$ we propose a novel in vitro assay named Quiescent-stage Survival Assay (QSA) to assess the efficacy of compounds against ARTs-induced quiescent parasites.

\section{MATERIALS AND METHODS}

\section{Parasite culture}

F32-ART5 (artemisinin-resistant), F32-TEM (artemisinin-sensitive) Plasmodium falciparum laboratory strains were cultivated at $37^{\circ} \mathrm{C}$ in RPMI-1640 medium (Biowest) at $2 \%$ hematocrit in human red blood cells and $5 \%$ AB human serum (EFS, French Blood Bank) under $5 \% \mathrm{CO}_{2}$ in a humid atmosphere at $37^{\circ} \mathrm{C} .{ }^{10,20,23,24}$ Artemisinin-resistant field isolates (from Pasteur Institute of Cambodia) IPC8262, IPC8317, IPC6388 and IPC6938 were cultivated under $5 \%$ $\mathrm{O}_{2}, 5 \% \mathrm{CO}_{2}$, and $90 \% \mathrm{~N}_{2}$ (FIVAL, Air Liquide) at $2 \%$ hematocrit in RMPI-1640 medium supplemented with $5 \%$ human serum (EFS, French Blood Bank) and $0.55 \%$ (w/w) albumax II (Thermo Fisher). ${ }^{25}$ The characteristics of the different strains used in this study are reported in Table 1. Genotyping of field isolates were given by Pasteur Institute of Cambodia.

\section{Drugs}

Chloroquine diphosphate, lumefantrine and mefloquine hydrochloride were purchased from Sigma-Aldrich (France), artemisinin from TCI Europe, atovaquone from GlaxoSmithKline, dihydroartemisinin, $\mathrm{N}$-desethyl amodiaquine and piperaquine tetraphosphate tetrahydrate were provided by the WWARN. Drugs were used in vitro in combination assays at pharmacologically relevant concentrations $(700 \mathrm{nM}$ dihydroartemisinin, $3 \mu \mathrm{M}$ artemisinin, 
$200 \mathrm{nM}$ chloroquine, $7 \mu \mathrm{M}$ atovaquone, $200 \mathrm{nM}$ piperaquine, $964 \mathrm{nM}$ mefloquine, $800 \mathrm{nM} \mathrm{N}$ desethyl amodiaquine, $5 \mu \mathrm{M}$ lumefantrine), equal or close to their plasma peak concentrations in patients. ${ }^{26}$ Stock solutions were obtained by dissolving piperaquine in $0.5 \%$ lactic acid (Sigma-Aldrich), $\mathrm{N}$-desethyl amodiaquine and chloroquine in RPMI-1640, while the other compounds were dissolved in DMSO (Sigma-Aldrich).

\section{Standard chemosensitivity assay}

The chemosensitivity assay was carried out in 96-well culture plates on D-sorbitol (SigmaAldrich) ring-stage synchronized parasites. ${ }^{27}$ The antiplasmodial activities were evaluated using the SYBR Green I method. ${ }^{28}$ Each drug concentration was tested in triplicates. The parasites were incubated with the drugs for $48 \mathrm{~h}$. Parasite pellets were then washed in 1X PBS prior to lysing red blood cells at $-20{ }^{\circ} \mathrm{C}$ overnight. Then, the plates were thawed and $100 \mu \mathrm{L}$ of each well were transferred into a black 96-well plate. $100 \mu \mathrm{L}$ of SYBR Green I (Thermo Fisher), diluted at a final concentration of $2 \mathrm{X}$ in lysis buffer, were added to each well and incubated for $1 \mathrm{~h}$ at room temperature prior to reading the plates on BioTek FLx 800 Microplate Fluorescence Reader $\left(\lambda_{\text {excitation }}=485 \mathrm{~nm}, \lambda_{\text {emission }}=528 \mathrm{~nm}\right) . \mathrm{IC}_{50}$ values were determined using GraphPad Prism software.

\section{Quiescent-stage Survival Assay (QSA)}

To evaluate drug activity in quiescent artemisinin-resistant parasites, the Quiescent-stage Survival Assay (QSA) was first developed using the F32-ART5 strain. D-sorbitol synchronized ring-form cultures at $3 \%$ parasitemia and $2 \%$ hematocrit $(5 \mathrm{~mL}$ of culture per well in 6 -well plate) were first exposed to dihydroartemisinin at $700 \mathrm{nM}$ for $6 \mathrm{~h}$ to induce quiescence of artemisinin-resistant parasites. Then parasite cultures were exposed to the combination, dihydroartemisinin + compound to be tested, for $48 \mathrm{~h}$ (Fig. 1A, condition a). Keeping parasites 
under dihydroartemisinin treatment during exposure to the compound to be tested ensured quiescence maintenance. In parallel, other combinations were tested as control conditions (Fig. 1A, conditions b-d). The compounds were also evaluated on proliferating artemisinin-resistant parasites i.e. without any pre-treatment (Fig. 1A, condition e). Between each drug treatment, parasite cultures were washed with $40 \mathrm{~mL}$ of RPMI-1640 medium and put back in culture in new wells for the next treatment. After drug exposure, parasite cultures were washed with $40 \mathrm{~mL}$ of RPMI-1640 medium and cultured in drug-free conditions supplemented with $10 \%$ human serum in new wells. The parasitemia for each condition was then microscopically monitored every 2 days to determine the time required to reach the initial parasitemia ( $3 \%)$, this time point being referred to as recrudescence day. Culture media were renewed twice a week and $25 \mu \mathrm{L}$ of fresh red blood cells were added once a week. If no recrudescence occurred after 30 days, the experiment was stopped. ${ }^{10,20}$ Delay in recrudescence time is the difference between the median recrudescence time from different independent experiments obtained with the drug combination (dihydroartemisinin $6 \mathrm{~h} /$ (dihydroartemisinin + drug) $48 \mathrm{~h}$ ) (Fig. 1A, condition a) and the median recrudescence time obtained with dihydroartemisinin alone (dihydroartemisinin $6 \mathrm{~h} /$ dihydroartemisinin $48 \mathrm{~h}$ ) (Fig. 1A, condition b). This delay is representative of the capacity of the tested compound to be active against quiescent parasites. Concentrations and treatment durations were chosen for their pharmacological relevance. During the QSA development, several conditions were tested to optimize the assay: i) 24-h or 48-h incubation of the compounds, ii) use of a physiological dose of $3 \mu \mathrm{M}$ of ART or $700 \mathrm{nM}$ of DHA to induce quiescence, iii) repetition of the drug combination treatments to mimic ACT pharmacology, and iv) 2 sorbitol treatments, at $30 \mathrm{~h}$ and $54 \mathrm{~h}$ during drug exposure to be sure that parasites were in a quiescent state.

\section{Recrudescence assay}


Recrudescence assay was carried out as previously reported to evaluate the ability of the F32-

ART5 parasite line to survive drug exposure comparatively to the artemisinin-sensitive F32TEM parasite line. ${ }^{10,20}$ After 48 -h drug exposure, synchronized ring-stage parasites were washed with RPMI-1640 medium and placed in normal drug-free culture conditions. The time to parasite recrudescence was determined as the time required to reach initial parasitemia. The experiment was stopped if no recrudescence was microscopically observed within 30 days. ${ }^{10,20}$

\section{Ring-stage Survival Assay (RSA)}

The Ring-stage Survival Assay (RSA) was carried out as previously reported. ${ }^{29}$ Briefly, 03 hours old ring-stage parasites (obtained after a Percoll® (Sigma-Aldrich)-sorbitol treatment) were exposed to $700 \mathrm{nM}$ DHA or $0.1 \%$ DMSO (control) for 6 hours in duplicate, then washed in RPMI-1640 and replaced in normal culture conditions for the next $72 \mathrm{~h}$. Survival rates were measured microscopically on Diff-Quick® (RAL Diagnostics)-stained thin blood smears. The parasitemia was calculated by counting 10,000 red blood cells per assay by two independent microscopists. A parasite growth rate $\geq 2$ in non-exposed culture was deemed necessary to validate the assay. The strains with a survival rate of dihydroartemisinin-treated parasites relative to control above $1 \%$ in RSA were referred to as artemisinin-resistant. ${ }^{29}$

\section{Piperaquine survival assay (PSA)}

The piperaquine survival assay (PSA) was performed as previously described to evaluate the ability of ring-stage parasites to survive piperaquine exposure. ${ }^{30}$ Briefly, $0-3 \mathrm{~h}$ old ring-stage parasites (obtained after a percoll-sorbitol treatment) were exposed to $200 \mathrm{nM}$ of piperaquine or culture medium alone (control) for $48 \mathrm{~h}$ in duplicate. After drug exposure, parasites were washed with RPMI-1640 medium and then cultivated for the next $24 \mathrm{~h}$ without drug. Survival rates were measured similarly to the RSA (ratio of viable parasites in piperaquine-exposed 
relative to DMSO-exposed samples). The strains with a survival rate above $10 \%$ in PSA were referred to as piperaquine-resistant. ${ }^{30}$

\section{Data Analysis}

Where relevant, a statistical analysis (log-rank test) was carried out on data from recrudescence assay and QSA by performing Kaplan-Meier survival analyses considering censored data. The outcome was defined as the time necessary for cultures to reach initial parasitemia. Observations were considered as censored if no recrudescence was observed within the experiment timeframe (30 days).

\section{RESULTS AND DISCUSSION}

The QSA (quiescent-stage survival assay), a relevant method to specifically evaluate chemosensitivity of quiescent parasites

Quiescence-based artemisinin resistance is a new challenge to consider when proposing relevant antiplasmodial treatments. In this context, we designed a novel assay, the QSA, to evaluate drug activity against artemisinin-resistant parasites in a quiescence state. The QSA interpretation is based on a delay in recrudescence time between cultures exposed to (dihydroartemisinin $6 \mathrm{~h} /$ (dihydroartemisinin + molecule) $48 \mathrm{~h}$ ) and cultures exposed to (dihydroartemisinin $6 \mathrm{~h} /$ dihydroartemisinin $48 \mathrm{~h}$ ) (Fig. 1A). A delayed recrudescence (Fig. 1B) or a lack thereof indicates that most or all quiescent parasites are killed by the partner drug tested. Therefore, it is suggested that the more potent the drug against quiescent parasites, the longer the delay because the fewer quiescent parasites, the slower the culture will grow back to initial parasitemia. According to the different results obtained and in order to select relevant compounds, we considered a molecule to be active against quiescent parasites if its recrudescence delay was above a 6-day threshold (Fig. 1B). Below this threshold, the difference 
could be statistically significant but not necessarily biologically relevant since this could be due to variations in culture parameters independent of the number of surviving parasites. Therefore, from one experiment to the other, there can be a two to three-day variation in recrudescence days, which is why, by setting a 6-day confidence interval, we made sure that the differences were meaningful. To develop the QSA model, chloroquine and atovaquone were used because of their different and well-documented mechanisms of action. The highly artemisinin-resistant P. falciparum strain F32-ART5 harboring none of the genotypic markers associated with chloroquine, mefloquine or atovaquone resistance was used (Table 1). ${ }^{10,20}$ Results were then confirmed on artemisinin-resistant isolates from the field.

\section{Chloroquine cannot kill quiescent parasites}

In the absence of dihydroartemisinin pre-treatment, F32-ART5 proliferative parasites appeared sensitive to a single dose of $200 \mathrm{nM}$ chloroquine for $48 \mathrm{~h}$ leading to parasite elimination with no recrudescence at day 30 (Fig. 2, Table S1). This chloroquine sensitivity of F32-ART5 is in accordance with the obtained $\mathrm{IC}_{50}$ values below the chloroquine-resistance cut-off of $100 \mathrm{nM}$ (Table S2). The combination dihydroartemisinin $6 \mathrm{~h} /$ chloroquine $48 \mathrm{~h}$ delays recrudescence time of resistant parasites by 7 days compared to dihydroartemisinin $6 \mathrm{~h}$ (Table S1). This delayed recrudescence time could be interpreted as the ability of chloroquine to either be active against quiescent parasites or to repress cell cycle recovery of quiescent parasites. To ensure the targeting of quiescent parasites, the quiescent state was maintained with dihydroartemisinin all over the chloroquine treatment. Surprisingly, the 2 conditions (dihydroartemisinin $6 \mathrm{~h} /$ (dihydroartemisinin + chloroquine) $48 \mathrm{~h}$ ) and (dihydroartemisinin $6 \mathrm{~h} /$ dihydroartemisinin $48 \mathrm{~h}$ ) showed similar recrudescence profiles (8 and 6 days, respectively) (Fig. 2, Table S1), which is interpreted as a lack of chloroquine activity against quiescent parasites. The mechanism of action of chloroquine, through the inhibition of hemozoin formation, could 
explain this result since such a mechanism is absent at the quiescent ring-stage. ${ }^{31} \mathrm{~A}$ recent study showed that chloroquine is active against both early ring stages and trophozoites, suggesting that hemoglobin digestion starts early on in the asexual cycle. ${ }^{32}$ Hence, this showcases that the efficacy of a drug against quiescent parasites cannot be deduced from its efficacy against proliferating parasites. The QSA condition (dihydroartemisinin $6 \mathrm{~h} /$ (dihydroartemisinin + drug to be tested) $48 \mathrm{~h}$ ) appears thus highly selective for identification of compounds genuinely active against quiescent forms. These results evidenced that drug activity differs according to the proliferating or quiescent state of parasites. Therefore, QSA appears as a global chemosensitivity assay that is able to evaluate drug activity against both artemisininresistant proliferating and quiescent parasites.

In order to better mimic the antimalarial drug regimens, the impact of repetitive chloroquine treatments was tested on quiescent parasites. Because a repeated (dihydroartemisinin $6 \mathrm{~h} /$ dihydroartemisinin $48 \mathrm{~h}$ ) treatment did not yield any recrudescence (data not shown), the $48 \mathrm{~h}$ exposure was thus reduced to $24 \mathrm{~h}$ in each cycle. In these conditions, two successive treatments (dihydroartemisinin $6 \mathrm{~h} /$ (dihydroartemisinin + chloroquine ) $24 \mathrm{~h} /$ dihydroartemisinin $6 \mathrm{~h} /$ (dihydroartemisinin + chloroquine) $24 \mathrm{~h}$ ) led to the same recrudescence time of 12 days as a single $60 \mathrm{~h}$ exposure to dihydroartemisinin alone (Table S3). This result was consistent with the total absence of chloroquine activity against quiescent parasites, independent of the treatment duration.

\section{Atovaquone targets both quiescent and proliferating parasites}

The delayed parasite recrudescence time of 12 days for the combination (dihydroartemisinin $6 \mathrm{~h} /$ (dihydroartemisinin + atovaquone) $48 \mathrm{~h}$ ) compared to dihydroartemisinin alone (Fig. 2, Table 2 and Table S4) evidenced that atovaquone is not only highly active against quiescent parasites but also against proliferating artemisinin-resistant parasites (Fig. 1, control condition 
e). This is in accordance with previously published atovaquone activity towards the

247 mitochondrial electron transport chain, ${ }^{33,34}$ whose activity seems maintained during quiescence. ${ }^{10,12,35}$

\section{The newly-designed QSA specifically targets quiescent parasites}

The induction of quiescence by ARTs had already been demonstrated. ${ }^{10}$ Here, two sorbitol treatments were performed during the QSA to eliminate any potential non ring-stage and thus non-quiescent parasites or awaking parasites (Fig. 3A). Regardless of the treatment conditions and the drugs used, sorbitol treatment did not lead to any delay in the recrudescence times compared to the no-sorbitol condition (Fig. 3B, Table S5). These data confirm that the QSA allows the specifically evaluation of the activities of drugs on $P$. falciparum quiescent parasites.

\section{QSA applied to artemisinin-resistant field isolates confirms its stage specificity}

The QSA was also performed on artemisinin-resistant isolates from the field to confirm its potential as a new screening tool for antimalarial development. Chloroquine and atovaquone were tested on four $P$. falciparum Cambodian isolates all harboring the C580Y mutation on the kelch13 gene and having survival rates above $1 \%$ in RSA, confirming their artemisinin resistance (Table 1). Notably, all the isolates used harbored a chloroquine-resistant phenotype with $\mathrm{IC}_{50}$ values superior to $100 \mathrm{nM}$ (Table S2). This phenotype was confirmed by the fast parasite recrudescence after 48 -h chloroquine treatment (2-day recrudescence time for IPC8262, IPC6938, IPC6388, and 6-day recrudescence time for IPC8317, compared to no recrudescence of the chloroquine-sensitive F32-ART strain) (Fig. 2, Table S1). Independent of the isolate used, the QSA confirmed the lack of chloroquine activity towards quiescent parasites with no significant delay between drug combination and the dihydroartemisinin control (Table 2, Table S1). 
The efficacy of atovaquone was confirmed regardless of the field isolate and the combination tested, with delays in recrudescence times all superior (at least 6 days) to the control condition (dihydroartemisinin $54 \mathrm{~h}$ ) supporting atovaquone activity against both quiescent (conditions ab) and artemisinin-resistant proliferating parasites (condition e) from the field (Fig. 2, Table 2, Table S4).

By validating the QSA protocol on both laboratory and field-isolated artemisinin-resistant strains, we evidenced that this newly designed protocol was able to evaluate the activity of compounds against artemisinin-resistant parasites both in the specific artemisinin-induced quiescent state and in proliferating forms.

\section{ACT partner drugs display different activities against artemisinin-resistant quiescent}

\section{parasites}

After standardizing the QSA protocol, the activity of WHO-recommended ACT partner drugs, namely $N$-desethyl amodiaquine (the active metabolite of amodiaquine), piperaquine, lumefantrine, and mefloquine were evaluated on quiescent parasites.

Although active towards proliferative parasites (Table S6, Fig. 1A condition e), $N$-desethyl amodiaquine showed a very weak activity against quiescent parasites from the laboratory strain F32-ART5 and the field isolate IPC8317 with a delay of 3-4 days (Fig. 2, Table S6), which is below the 6-day delay threshold. This lack of efficacy towards quiescent parasites could be explained by the mode of action of the molecule which, like chloroquine, ${ }^{36,37}$ targets the polymerization of heme into hemozoin, a mechanism absent during quiescence. This is further corroborated by the stage specificity of $N$-desethyl amodiaquine, which is mostly active against trophozoites. ${ }^{38}$

In a similar fashion, a weak piperaquine activity was observed in QSA against quiescent parasites with a delay in recrudescence time below the 6-day threshold for F32-ART5, 
IPC8317, IPC8262 and IPC6388, (Fig. 2, Table 2, Table S7). These strains harbor a single copy of the pm2 gene (except for IPC8262 with $1.58 \mathrm{pm} 2$ copies) and a PSA survival rate below $10 \%$ (Table 1). Firstly, the absence of delay between F32-ART and F32-TEM in piperaquine recrudescence assay and PSA data confirm that the partial lack of piperaquine activity against quiescent parasites is not linked with any piperaquine resistance or cross-resistance with artemisinin (Table S8). Secondly, these results are in accordance with the purported mode of action of piperaquine which is thought to interfere with heme detoxification in a similar fashion and with similar stage specificity than chloroquine. ${ }^{32,39}$

The field isolate IPC6938 showed no recrudescence after treatment with piperaquine alone whereas this line harbors a PSA survival rate of $10.7 \%$ and 3 copies of the $p m 2$ gene consistent with piperaquine resistance (Table 1). These conflicting results could be explained by the age of ring-stage parasites during drug exposure in the different assays. Indeed, when PSA was performed on ring-stage parasites older than 6 hours (condition similar to the QSA), the survival rate dropped to $0.16 \%$. Unlike the other parasite lines, preliminary data obtained with this isolate showed a delayed recrudescence with piperaquine above 6 days in QSA (Table 2, Table S7). Further experiments should be conducted in order to clarify the impact of piperaquine resistance on quiescent parasites.

In contrast, lumefantrine appeared active against quiescent parasites both on the laboratory strain F32-ART5 and on the parasite isolate from the field IPC8317 with a recrudescence time delay above 9 days (Fig. 2, Table 2, Table S9). Recently, lumefantrine has been shown to be active against all but the schizont stage. ${ }^{32}$ These data, in addition to our results which also showed its activity against quiescent parasites, seem to indicate that lumefantrine targets other pathway(s) apart from the inhibition of hemozoin formation. ${ }^{40}$

Mefloquine also showed an activity against quiescent parasites on all the tested strains with very high recrudescence delays (Fig. 2, Table 2, Table S10). One field isolate, IPC6388, showed 
a faster recrudescence after treatment with mefloquine alone compared to the other strains, correlating with a higher $\mathrm{IC}_{50}$ value. Consistently, this isolate harbors 3 copies of the $m d r 1$ gene which is associated with mefloquine resistance (Table 1). Interestingly, its recrudescence delay was of 6 days in QSA. Therefore, these results suggest that although mefloquine-resistant parasites in quiescent state are less sensitive to the drug than mefloquine-sensitive parasites, they can still be killed by mefloquine treatment. Its activity against quiescent parasites is surprising considering its specificity towards trophozoites. ${ }^{32}$ It cannot be explained by its ability to impair hemoglobin uptake, ${ }^{41}$ hemozoin biosynthesis, ${ }^{42}$ or even inhibit protein synthesis by targeting the $80 \mathrm{~S}$ ribosome, ${ }^{43}$ since these different pathways are supposed to be inactive during quiescence. Although the effect of dihydroartemisinin treatment on metacaspase transcripts remains to be determined, ${ }^{44}$ the mefloquine activity against quiescent parasites could be due to its capacity to trigger an apoptotic process by Plasmodium metacaspase activation leading to parasite death. ${ }^{45,46}$

\section{Targeting quiescent parasites can be achieved with different treatment schedules}

In order to optimize the QSA protocol, different quiescence-inducing drugs and different drug schedules were tested against F32-ART5 in combination with 4 antimalarials namely chloroquine, atovaquone, piperaquine and mefloquine. Similar activities were obtained regardless of the artemisinin derivative used $(3 \mu \mathrm{M}$ of artemisinin or $700 \mathrm{nM}$ of dihydroartemisinin) or the treatment duration ( $24 \mathrm{~h}$ or $48 \mathrm{~h}$; Table S11) except when treating quiescent parasites with piperaquine. Indeed, a 24-h exposure to piperaquine has no effect on quiescent parasites as shown by the lack of delay between the combination and the control regardless of the drug used to induce quiescence (Table S11), whereas a 48-h exposure to piperaquine led to a 5-day delay (Table 2). Because ACT partner drugs have half-lives of several days, we reasoned that lengthening drug exposure to 48 hours (corresponding to a 
hypothetical complete erythrocytic cycle) allowed better approximation of physiological conditions.

Tests of molecules against quiescent parasites had already been reported. ${ }^{11,47-49}$ However, our results underlined the importance of i) pre-treatment by ARTs to induce quiescence, ii) maintaining quiescence throughout the whole drug evaluation, and iii) comparing the combination dihydroartemisinin / (dihydroartemisinin + drug to be tested) to dihydroartemisinin / dihydroartemisinin in order to be able to conclude on the genuine efficacy of the drug towards quiescent parasites.

\section{Utility and limitations of the QSA}

Standard chemosensitivity assays are proliferation-based and hence, cannot allow the evaluation of the missing variable of partner drug activity against quiescent parasites. Because of its experimental design, the QSA presented herein can overcome these limitations. This assay appears as a highly relevant tool to study artemisinin-induced quiescence in $P$. falciparum parasites. It also constitutes an in vitro model that tries to grasp the pharmacological complexity of ACTs by triggering quiescence before exposing parasites to the partner drug. Compounds tested were used at high concentrations over 48 hours to approximate their physiological conditions since ACT partner drugs have half-lives of several days and high plasma peak concentrations. ${ }^{26}$ Therefore, these parameters could be fine-tuned when evaluating the activity of other compounds. The assay was not designed to compare the activity of different drugs on quiescent parasites but rather to determine whether a drug efficiently kills quiescent parasites.

367 Moreover, it does not allow direct assessment of parasite death for obvious quantitation 368 limitations. The outcome of the assay is thereby indirect as it relies on a delayed parasite recrudescence, correlated with the ability of a drug to kill quiescent parasites. 
In conclusion, thanks to the QSA, we highlighted that i) drug activity against quiescent parasites depends on the metabolic status of its target throughout quiescence, regardless of the drug's stage specificity in asexual replication, ii) because of quiescence-based mechanism, drug efficacy against artemisinin-induced quiescent forms of the falciparum malaria parasite is unrelated to its activity against proliferating parasites, and iii) some molecules active against ARTs-induced quiescent parasites can be identified and can shed light on pathways that remain active within this dormant state, potentially guiding the search for new antimalarials. Custom ACTs through the addition of a third compound are already evaluated in clinical trials. ${ }^{50}$ However the added value of QSA-based drug design would be to enable the design of novel ACTs that effectively target dormant parasites. This strategy could greatly enhance ACT clinical efficacy and drastically limit the emergence of resistant parasites. Ultimately, this approach could also confer an extended lifespan to ACTs even in a drug resistance context.

\section{Acknowledgements}

We would like to thank Chinedu O. Egwu for proofreading the article and we would also like to acknowledge the WorldWide Antimalarial Resistance Network (WWARN) Reference Standards Program for reference drug providing.

\section{Funding}

This study was supported in part by the Centre National de la Recherche Scientifique and the French Agence Nationale de la Recherche (ANR-13-BSV3-0018 \& ANR-13-CE35-0003). T. R. was funded by a Graduate Fellowship Program from the University Paul Sabatier of Toulouse. The funding sources of the study had no role in the study design, data collection, data analysis, data interpretation or writing of the report. 
396 Transparency declarations

397 None to declare.

398 


\section{References}

400 1. White N. Antimalarial drug resistance and combination chemotherapy. Philos Trans R Soc B Biol $401 \quad$ Sci 1999; 354: 739-49.

402 2. World Health Organization. World Malaria Report 2019. 2019.

403 https://www.who.int/publications-detail/world-malaria-report-2019

404 3. Noedl H, Se Y, Schaecher K et al. Evidence of artemisinin-resistant malaria in Western Cambodia. $405 \quad$ N Engl J Med 2008; 359: 2619-20.

406 4. Dondorp AM, Nosten F, Yi P et al. Artemisinin Resistance in Plasmodium falciparum Malaria. $N$ 407 Engl J Med 2009; 361: 455-67.

408 5. Ashley EA, Dhorda M, Fairhurst RM et al. Spread of Artemisinin Resistance in Plasmodium 409 falciparum Malaria. N Engl J Med 2014; 371: 411-23.

410 6. Ménard D, Khim N, Beghain J et al. A worldwide map of Plasmodium falciparum K13-propeller 411 polymorphisms. N Engl J Med 2016; 374: 2453-64.

7. World Health Organization. Status report on Artemisinin and ACT resistance (April 2017). 2017. http://www.who.int/malaria/publications/atoz/artemisinin-resistance-april2017/en/ 8. Ariey F, Witkowski B, Amaratunga C et al. A molecular marker of artemisinin-resistant Plasmodium falciparum malaria. Nature 2014; 505: 50-5.

9. Birnbaum J, Scharf S, Schmidt S et al. A Kelch13-defined endocytosis pathway mediates artemisinin resistance in malaria parasites. Science 2020; 367: 51-59. 10. Witkowski B, Lelièvre J, Barragán MJL et al. Increased tolerance to artemisinin in Plasmodium falciparum is mediated by a quiescence mechanism. Antimicrob Agents Chemother 2010; 54: 1872-7. 11. Chen N, LaCrue AN, Teuscher F et al. Fatty Acid Synthesis and Pyruvate Metabolism Pathways Remain Active in Dihydroartemisinin-Induced Dormant Ring Stages of Plasmodium falciparum. Antimicrob Agents Chemother 2014; 58: 4773-81. 12. Paloque L, Ramadani AP, Mercereau-puijalon O et al. Plasmodium falciparum: multifaceted resistance to artemisinins. Malar J 2016; 15: 149. 13. Witkowski B, Khim N, Chim P et al. Reduced artemisinin susceptibility of Plasmodium falciparum ring stages in western cambodia. Antimicrob Agents Chemother 2013; 57: 914-23. 

border: integration of clinico-parasitological response, systemic drug exposure, and in vitro parasite sensitivity. Malar J 2013; 12: 263. 15. Saunders DL, Vanachayangkul P, Lon C. Dihydroartemisinin-piperaquine failure in Cambodia. $N$ Engl J Med 2014; 371: 484-5.

16. Spring MD, Lin JT, Manning JE et al. Dihydroartemisinin-piperaquine failure associated with a triple mutant including kelch13 C580Y in Cambodia: An observational cohort study. Lancet Infect Dis 2015; 15: 683-91.

17. Dondorp AM, Fairhurst RM. Artemisinin-Resistant Plasmodium falciparum Malaria. Microbiol Spectr 2016; 4: 409-29.

18. Amaratunga C, Lim P, Suon S et al. Dihydroartemisinin-piperaquine resistance in Plasmodium falciparum malaria in Cambodia: a multisite prospective cohort study. Lancet Infect Dis 2016; 16: $357-65$.

19. Amato R, Pearson RD, Almagro-Garcia $\mathbf{J}$ et al. Origins of the current outbreak of multidrugresistant malaria in southeast Asia: a retrospective genetic study. Lancet Infect Dis 2018; 18: 337-45. 20. Ménard S, Ben Haddou T, Ramadani AP et al. Induction of multidrug tolerance in Plasmodium falciparum by extended artemisinin pressure. Emerg Infect Dis 2015; 21: 1733-41. falciparum in response to artemisinin combination therapies in Thailand. Clin Microbiol Infect 2016; 22: $285 . e 1-8$.

22. WHO. Status report on artemisinin resistance. 2014. https://www.who.int/malaria/publications/atoz/status-rep-artemisinin-resistance-sep2014.pdf 23. Benoit-Vical F, Lelièvre J, Berry A et al. Trioxaquines are new antimalarial agents active on all erythrocytic forms, including gametocytes. Antimicrob Agents Chemother 2007. 51: 1463-72 24. Wenger RH, Kurtcuoglu V, Scholz CC et al. Frequently asked questions in hypoxia research. Hypoxia (Auckland, NZ) 2015; 3: 35-43. 
evaluation of malian Plasmodium falciparum isolates. Am J Trop Med Hyg 2018; 98: 1123-31.

26. Nosten F, White NJ. Artemisinin-based combination treatment of falciparum malaria. Am J Trop Med Hyg 2007; 77: 181-92.

27. Lambros C, Vanderberg JP. Synchronization of Plasmodium falciparum Erythrocytic Stages in Culture. J Parasitol 1979; 65: 418-20.

28. Smilkstein M, Sriwilaijaroen N, Kelly JX et al. Simple and Inexpensive Fluorescence-Based Technique for High-Throughput Antimalarial Drug Screening. Antimicrob Agents Chemother 2004; 48: $1803-6$.

29. Witkowski B, Amaratunga C, Khim N et al. Novel phenotypic assays for the detection of artemisinin-resistant Plasmodium falciparum malaria in Cambodia: In-vitro and ex-vivo drug-response studies. Lancet Infect Dis 2013; 13: 1043-9.

30. Duru V, Khim N, Leang R et al. Plasmodium falciparum dihydroartemisinin-piperaquine failures in Cambodia are associated with mutant K13 parasites presenting high survival rates in novel piperaquine in vitro assays: Retrospective and prospective investigations. BMC Med 2015; 13: 305. 31. Fitch CD. Ferriprotoporphyrin IX, phospholipids, and the antimalarial actions of quinoline drugs. Life Sci 2004; 74: 1957-72.

32. Murithi JM, Owen ES, Istvan ES et al. Combining Stage Specificity and Metabolomic Profiling to Advance Antimalarial Drug Discovery. Cell Chem Biol 2020; 27: 158-171.e3.

33. Nixon GL, Pidathala $\mathrm{C}$, Shone AE et al. Targeting the mitochondrial electron transport chain of Plasmodium falciparum: New strategies towards the development of improved antimalarials for the elimination era. Future Med Chem 2013; 5: 1573-91.

34. Siregar JE, Kurisu G, Kobayashi T et al. Direct evidence for the atovaquone action on the Plasmodium cytochrome bc1 complex. Parasitol Int 2015; 64: 295-300.

35. Peatey CL, Chavchich M, Chen N et al. Mitochondrial Membrane Potential in a Small Subset of Artemisinin-Induced Dormant Plasmodium falciparum Parasites in Vitro. J Infect Dis 2015. 212: 42634

36. Bray PG, Mungthin M, Ridley RG et al. Access to Hematin: The Basis of Chloroquine Resistance. Mol Pharmacol 1998; 54: 170-9. 
37. Ginsburg H, Famin O, Zhang J et al. Inhibition of glutathione-dependent degradation of heme by chloroquine and amodiaquine as a possible basis for their antimalarial mode of action. Biochem Pharmacol 1998; 56: 1305-13.

38. Wilson DW, Langer C, Goodman CD et al. Defining the timing of action of antimalarial drugs against Plasmodium falciparum. Antimicrob Agents Chemother 2013; 57: 1455-67.

39. Sachanonta N, Chotivanich K, Chaisri U et al. Ultrastructural and real-time microscopic changes in P. falciparum-infected red blood cells following treatment with antimalarial drugs. Ultrastruct Pathol 2011; 35: 214-25.

40. Warhurst DC, Adagu IS, Beck H-P et al. Mode of action of artemether/lumefantrine (COARTEM): The sole, fixed, oral ADCC and its role in combatting multidrug-resistance. Southeast Asian J Trop Med Public Health 2001; 32: 4-8.

41. Famin O, Ginsburg H. Differential effects of 4-aminoquinoline-containing antimalarial drugs on hemoglobin digestion in Plasmodium falciparum-infected erythrocytes. Biochem Pharmacol 2002; 63: $393-8$.

42. Sullivan DJ, Matile H, Ridley RG et al. A common mechanism for blockade of heme polymerization by antimalarial quinolines. J Biol Chem 1998; 273: 31103-7.

43. Wong W, Bai XC, Sleebs BE et al. Mefloquine targets the Plasmodium falciparum 80S ribosome to inhibit protein synthesis. Nat Microbiol 2017; 2: 17031

44. Shaw PJ, Chaotheing S, Kaewprommal P et al. Plasmodium parasites mount an arrest response to dihydroartemisinin, as revealed by whole transcriptome shotgun sequencing (RNA-seq) and microarray study. BMC Genomics 2015; 16: 830.

45. Gunjan S, Singh SK, Sharma T et al. Mefloquine induces ROS mediated programmed cell death in malaria parasite: Plasmodium. Apoptosis 2016; 21: 955-64.

46. Meslin B, Beavogui AH, Fasel N et al. Plasmodium falciparum metacaspase PfMCA-1 triggers a z-VAD-fmk inhibitable protease to promote cell death. PLoS One 2011; 6: e23867.

47. Teuscher F, Gatton ML, Chen N et al. Artemisinin-Induced Dormancy in Plasmodium falciparum: Duration, Recovery Rates, and Implications in Treatment Failure. J Infect Dis 2010; 202: 1362-8. 
511 Rings in Wild-Type and K13 Artemisinin-Resistant Plasmodium falciparum in vitro. Antimicrob

512 Agents Chemother 2018; 62: e02235-17.

513 49. Clements RL, Streva V, Dumoulin P et al. A novel antiparasitic compound kills ring-stage

514 Plasmodium falciparum and retains activity against artemisinin-resistant parasites. J Infect Dis 2020;

$515 \quad 221: 956-62$

516 50. Thu AM, Phyo AP, Landier J et al. Combating multidrug-resistant Plasmodium falciparum

517 malaria. FEBS J 2017; 284: 2569-78.

518 


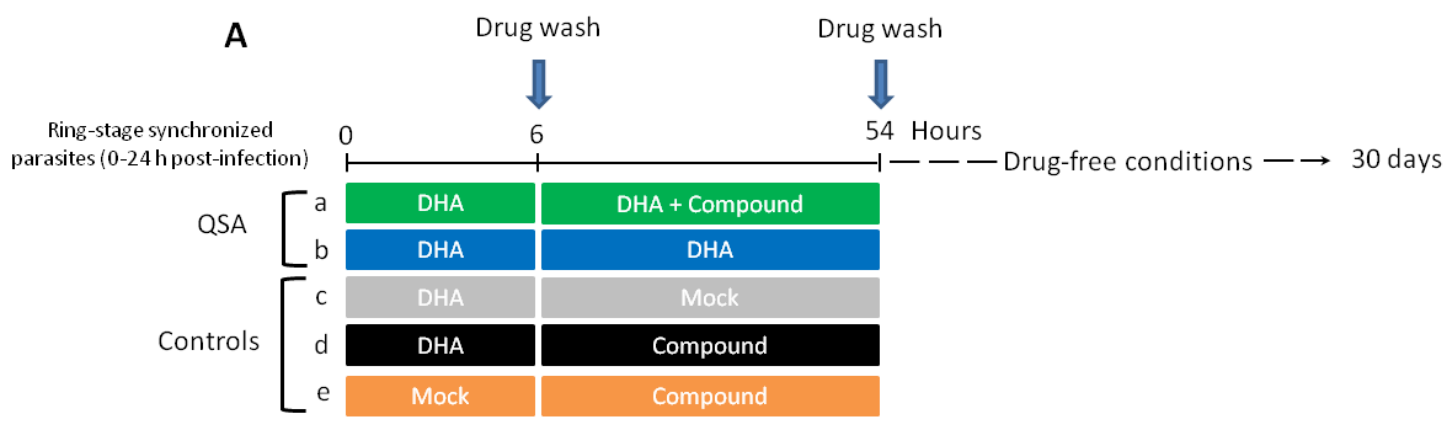

B Delays in recrudescence time

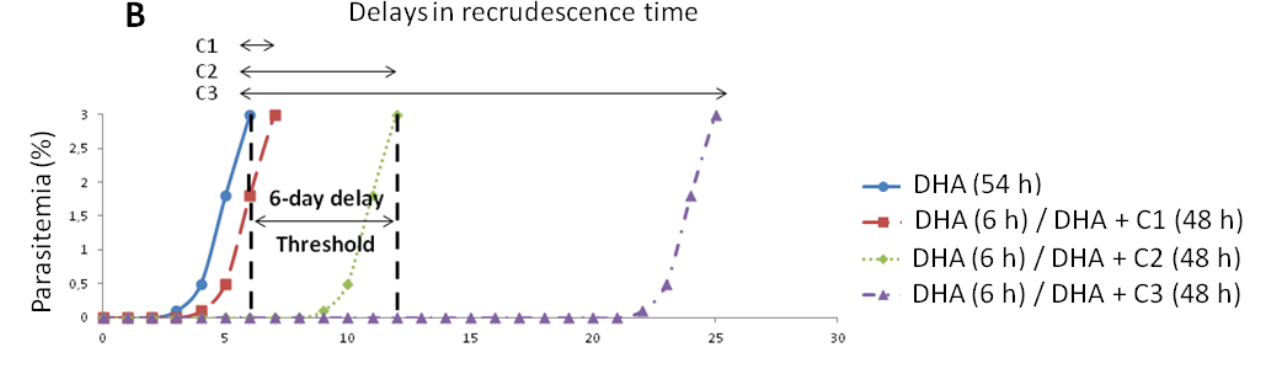

523 Figure 1. QSA (Quiescent-stage Survival Assay) design and interpretation. A: Treatment schedule of the different drug combinations studied (DHA: dihydroartemisinin); a-b) conditions to assess activity of the molecule on quiescent parasites; c-e) control conditions. B: Schematic curves of recrudescence profiles of artemisinin-resistant parasites to reach the initial $3 \%$ 527 parasitemia obtained via the QSA after 54-h treatment with DHA $700 \mathrm{nM}$ alone or in combination with different compounds tested $(\mathrm{C} 1, \mathrm{C} 2$ and $\mathrm{C} 3) . \mathrm{C} 1$ appears inactive, $\mathrm{C} 2$ is active with a 6-day delay (corresponding to the designated threshold) between $\mathbf{a}$ and $\mathbf{b}$ conditions and threshold. Double-headed arrows highlight delay in recrudescence times between combination treatments (DHA / DHA $+\mathrm{C} 1, \mathrm{C} 2$ or C3) and DHA (54 h) alone. 


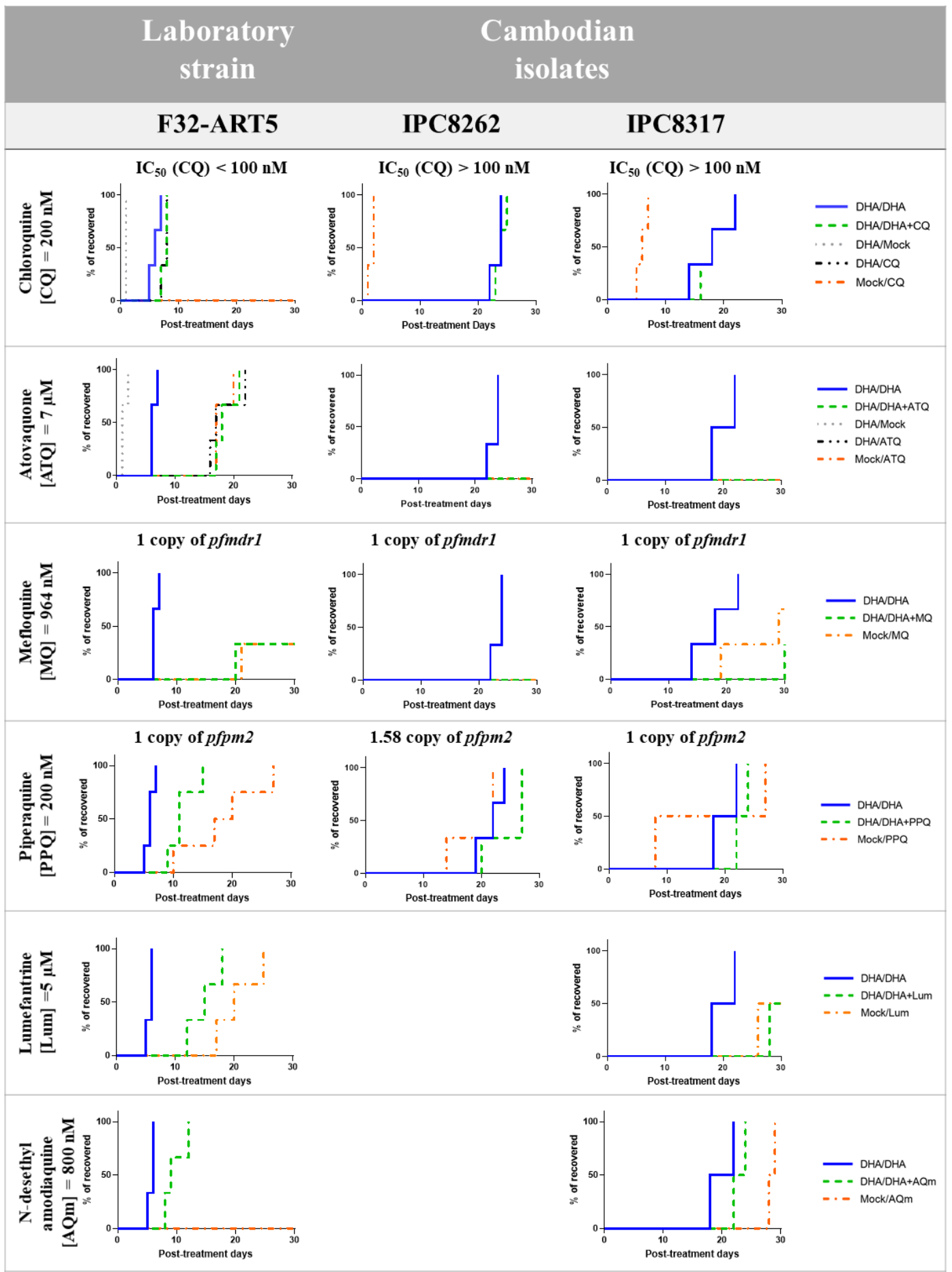

535 Figure 2. QSA (Quiescent-stage Survival Assay) results represented by Kaplan-Meier survival 536 curves showing recrudescence days of synchronous ring-stage parasites of the laboratory 537 artemisinin-resistant strain F32-ART5 and two artemisinin-resistant Cambodian isolates after 
538 different treatments. D0 corresponds to the day when all drugs were removed. Raw data are

539 presented in supplementary tables. These 3 strains are artemisinin-resistant (RSA survival 540 rates $>1 \%$ ), piperaquine-sensitive (PSA survival rates $<10 \%$ ) and mefloquine-sensitive. 
A

Ring-stage synchronized parasites (0-6 h post-infection)

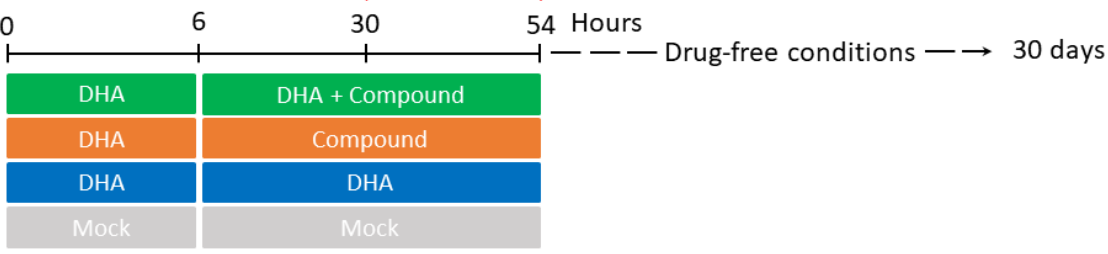

B

Chloroquine
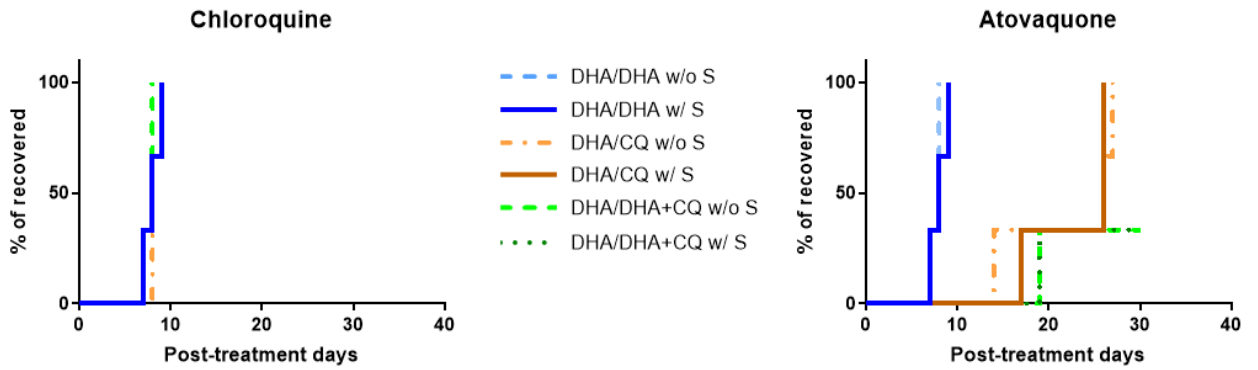

DHA/DHA w/o S

DHA/DHA w/S

-. DHA/ATQ W/o S

- DHA/ATQ w/S

- - DHAIDHA+ATQ w/o S

.... DHA/DHA+ATQ w/S

541

542

543

544

545

546

547 possible forms older than $24 \mathrm{~h}$ post-invasion preventing any recrudescence. Comparing the

548 effect of sorbitol treatment for a given treatment schedule will thus reveal if the QSA protocol

549 is indeed adequate to target quiescent forms of the parasite.

550

551

552 presented in supplementary Table S5.

553

w/ S (with sorbitol), w/o S (without sorbitol). 
Table 1. Phenotypic and genotypic traits of the P. falciparum strains used in this study

\begin{tabular}{|c|c|c|c|c|c|c|c|}
\hline \multirow[b]{2}{*}{ Strain } & \multirow{2}{*}{ Description } & \multicolumn{4}{|c|}{ Genotype } & \multirow{2}{*}{$\begin{array}{l}\text { RSA survival } \\
\text { rate }(\%)\end{array}$} & \multirow{2}{*}{$\begin{array}{l}\text { PSA survival } \\
\text { rate }(\%)^{\mathbf{b}}\end{array}$} \\
\hline & & pfkelch13 & pfcrt & pfpm 2 copy number & pfmdrl copy number & & \\
\hline F32-ART5 & Laboratory & M476I & WT & 1 copy & 1 copy & $10.7^{\mathrm{a}}$ & 0.4 \\
\hline F32-TEM & Laboratory & WT & WT & 1 copy & 1 copy & $0.09^{\mathrm{a}}$ & ND \\
\hline IPC8262 & Field & $\mathrm{C} 580 \mathrm{Y}$ & ND & 1.58 copy & 1 copy & $13^{b}$ & 2.7 \\
\hline IPC8317 & Field & C580Y & ND & 1 copy & 1 copy & $8.8^{c}$ & 0 \\
\hline IPC6388 & Field & C580Y & ND & 1 copy & 3 copies & $6.8^{\mathrm{c}}$ & 1.9 \\
\hline IPC6938 & Field & C580Y & ND & 3 copies & 1 copy & $6.6^{\mathrm{c}}$ & 10.7 \\
\hline
\end{tabular}

557 


\begin{tabular}{|c|c|c|c|c|c|c|}
\hline & \multicolumn{6}{|c|}{ Delay in recrudescence time (post-treatment days) } \\
\hline & $\mathbf{C Q}$ & ATQ & AQm & PPQ & LUM & MQ \\
\hline F32-ART5 & 2 & 12 & 4 & 5 & 10 & $>24$ \\
\hline IPC8262 & 0 & $>6$ & & 5 & & $>6$ \\
\hline IPC8317 & 0 & $>10$ & 3 & 3 & $>9$ & $>12$ \\
\hline IPC6938 & 3 & $>6$ & & $>6$ & & $>6$ \\
\hline IPC6388 & 0 & 14 & & 3 & & 6 \\
\hline
\end{tabular}

Table 2. Difference in median recrudescence times from independent experiments obtained for the artemisinin-resistant strain F32-ART5 and 4 Cambodian ART-resistant isolates following exposure to different antimalarial drugs in QSA conditions (DHA $6 \mathrm{~h} /$ (DHA + compound $48 \mathrm{~h}$ )) compared to DHA alone (DHA $6 \mathrm{~h} / \mathrm{DHA} 48 \mathrm{~h}$ ). Raw data are presented in supplementary tables. 70
571

572

573

574

575

576

577

578

579 